\title{
Effects of housing density on Long Evans and Fischer 344 rats
}

\author{
Krystal Bean, DVM ${ }^{1}$, Kevin Nemelka, DVM ${ }^{1}$, Patrick Canchola, DVM ${ }^{1}$, Sander Hacker, DVM², \\ Rodney X. Sturdivant, $P h D^{3}$ \& Pedro J. Rico, $D V M^{1}$
}

At many breeding facilities, rats are housed at relatively high densities until they are 5 weeks old, at which point they are either shipped for research or rehoused at standard cage densities according to weight. The authors carried out a pilot study in Long Evans and in Fischer 344 rats to investigate whether continuing to house rats at high densities (24 in ${ }^{2}$ floor space per rat) past the age of 5 weeks, through puberty and into adulthood would alter behavioral or physiological parameters compared with raising rats at standard densities (about 72 in $^{2}$ floor space per rat). After rats reached puberty, the authors rehoused them with unfamiliar cagemates. The researchers evaluated clinical and behavioral signs of stress, weight, blood glucose concentration, white blood cell count and serum corticosterone concentration. Overall, cage density had little effect on the parameters measured, though gender seemed to affect stress in Long Evans rats. The results suggest that rats of these strains can be raised at the higher densities tested until any age and regrouped with unfamiliar cagemates without compromising rats' welfare or subsequent experimental data.

The Guide for the Care and Use of Laboratory Animals (the Guide) provides cage space requirements for rats according to weight (Table 1). The Guide states that its space requirements may be adjusted for specific research protocols and in accordance with the compatibility of individual animals, as long as there is no risk to the animals' well-being ${ }^{1}$.

At the production facility from which we acquire rodents for research, young rats are housed in less than the recommended amount of floor space per rat from weaning age until they are about 5 weeks old (Technical Services Department, Charles River Laboratories, Wilmington, MA; personal communication). Most rats are then either shipped for use in research or rehoused in cages that meet the Guide's floor space recommendations.

We carried out a pilot study in which we housed rats at high densities between the ages of 5 weeks and 12-13 weeks. After rats reached puberty, we regrouped them with unfamiliar rats and continued to house them at high densities. We examined whether this process would alter behavioral or physiological characteristics in these rats compared with rats that were raised at standard housing densities. Lack of effect would indicate that rats may be raised at high densities until any age and regrouped with unfamiliar cagemates without compromising their welfare or data obtained in subsequent short-duration studies.

Numerous studies have evaluated stress in rodents by assessing behavior, weight change, food and water consumption and survival, as well as physiologic parameters such as heart rate, temperature, blood pressure, white blood cell count, blood glucose concentration and serum corticosterone concentration ${ }^{2-16}$. In this study, we examined rats for clinical and behavioral signs of stress (chromodacryorrhea, hair loss, wounds, inappetence, lethargy, ruffled haircoat and hunched appearance) that could make them unsuitable for research. We also tracked weight, blood glucose concentration, white

${ }^{1}$ Division of Veterinary Medicine, Walter Reed Army Institute of Research/Naval Medical Research Center, Silver Spring, MD.

${ }^{2}$ Department of Animal Medicine, University of Texas Health Sciences Center, San Antonio, TX. ${ }^{3}$ Department of Mathematics Sciences, United States Military Academy, West Point, NY. Correspondence should be addressed to K.B. (krystal.bean@us.army.mil). 
blood cell (WBC) count and serum corticosterone concentration. Changes to these parameters might indicate distress that is not evident by observation and that could affect research data collected from rats.

Previous studies have evaluated the effects of housing density on young mice or rats but have not continued to investigate whether these animals would be useful for research purposes if regrouped with unfamiliar animals at an older age $\mathrm{e}^{2,4,8,9,11,12,17-19}$. One group of researchers reported that housing post-pubertal Sprague Dawley rats at high densities resulted in decreased muscle mass in males but not in females ${ }^{20}$. In another study, 56-d-old Sprague Dawley rats that were arbitrarily grouped at high densities ( 3 rats per cage) showed no significant differences in fighting frequency or weight gain compared with rats that were housed singly; in addition, grouphoused rats had lower blood pressure at night ${ }^{3}$. In several studies of adult rats that were housed at high densities with unfamiliar rats, researchers observed behavioral changes associated with fighting and dominance in males, though not in females ${ }^{17,21-23}$. Studies that evaluated social behaviors related to fighting, dominance and territorial aggression reported that male rats older than $100 \mathrm{~d}$ showed territorial aggression and social dominance, whereas juvenile rats only showed play-fighting behavior $^{21-26}$. Another researcher showed that in male Wistar rats, the behavioral and physiological effects of high-density housing were dependent on the developmental stage of the rats ${ }^{27}$. These reports indicate that both gender and age may affect behavioral and physiological parameters in rats housed at high densities.

In this study, we examined male and female rats from two strains that are commonly used in research, the outbred Long Evans (LE) strain and the inbred Fischer 344 (F344) strain. The results of this study might be helpful in defining space recommendations for LE and F344 rats.

\section{METHODS}

\section{Rats and initial housing conditions}

The protocol in this experiment was reviewed and approved by the IACUC of Walter Reed Army Institute of Research/Naval Medical Research Center (WRAIR/NMRC). All procedures were carried out in a facility accredited by AAALAC, International.

We obtained LE ( $n=28 ; 14$ males and 14 females) and F344 rats ( $n=32 ; 16$ males and 16 females) from Charles River Laboratories (Wilmington, MA). At the breeding facility, LE rats were housed in groups of 40 in cages with total floor space of 24 in $\times 24$ in (14.4 in ${ }^{2}$ per rat). F344 rats were housed in groups of 30 in cages with total floor space of 13.88 in $\times 22.63$ in (10.47 in $^{2}$ per rat). Rats were housed in these conditions from weaning until they were 5 weeks old (Technical Services Department, Charles River Laboratories, Wilmington, MA; personal communication). These floor space allocations are slightly smaller than those recommended

TABLE 1 | The Guide's minimum floor space
recommendations for rats, according to weight
$\begin{array}{ll}\text { Rat weight }(\mathrm{g}) & \text { Floor space per rat } \\ <100 & 17 \mathrm{in}^{2} \\ 100-200 & 23 \mathrm{in}^{2} \\ 200-300 & 29 \mathrm{in}^{2} \\ 300-400 & 40 \mathrm{in}^{2} \\ 400-500 & 60 \mathrm{in}^{2} \\ >500 & \geq 70 \mathrm{in}^{2}\end{array}$

in the Guide for rats weighing $<100 \mathrm{~g}\left(17 \mathrm{in}^{2} \text { per rat }\right)^{1}$. At Charles River Laboratories, rats younger than 5 weeks are commonly housed at higher densities without high frequency of wounds, failure to thrive or other visible stress reactions. At 5 weeks of age, rats are either shipped to research facilities or housed according to the Guide's current space recommendations.

When they arrived at our facility, the F344 rats were 35-42 d old and the LE rats were 30-36 d old. Rats were free of Sendai virus, pneumonia virus of mice, coronavirus, Kilham's rat virus, Toolan's $\mathrm{H}-1$ virus, rat parvovirus, rat minute virus, reovirus, Mycoplasma pulmonis, lymphocytic choriomeningitis virus, hantavirus, ciliaassociated respiratory bacillus, Salmonella spp., Shigella spp., Leptospira spp., Spirillum minus, Helicobacter spp. and ectoparasites. We continued to monitor rats with sentinel testing throughout all stages of research.

\section{First stage: housing density study}

Rats arrived at our facility weighing 75-110 g each. Rats were ear-tagged for identification and then used in a separate study in which they were housed for 8 weeks at either a high density (5-7 rats per cage; $20.5-28$ in $^{2}$ floor space per rat) or a low density (2 rats per cage; 72 in $^{2}$ per rat). Rats were separated by gender and strain. For high-density housing, LE and F344 rats were housed, respectively, at 2-3 times and 1.1-1.4 times less space per rat than what is recommended in the Guide. For low-density housing, the amounts of floor space per rat for both strains were equal to or greater than the Guide's standard allowances.

During this stage of the study, all rats were subjected to similar manipulations and data collection procedures. None of the rats showed evidence of dominant or submissive behavior such as biting or barbering (excessive grooming). Each rat was within the expected weight range for its age, strain and gender ${ }^{28}$. No other signs of excessive stress or aggression, including inappetence, hunched posture or lethargy, were observed in any of the rats. Chromodacryorrhea was noted in some of the rats during this stage of the study.

\section{Second stage: regrouping with unfamiliar rats}

We began the second stage of research when rats were $12-13$ weeks old. The average age of puberty for rats is 
$50 \pm 10$ d (7-9 weeks; ref. 29). At this stage of the study, no rats were actively showing any unusual clinical signs.

We redistributed the groups of rats and rehoused them such that no rats that were previously housed together were assigned to the same cage. Rats that had been housed at a high density during the first stage of the study continued to be housed at a higher density than recommended (these rats are hereafter referred to as 'HDH'), and rats that had been housed at a low density continued to be housed in an amount of space that was equal to or greater than that recommended in the Guide (hereafter referred to as 'LDH'). We separated rats by gender and strain; otherwise, the selection of groups was arbitrary. All rats were subjected to the same manipulations and data collection procedures.

We housed LE rats in groups of six for HDH (24 in ${ }^{2}$ floor space per rat) and in groups of two for $\mathrm{LDH}$ (72 in ${ }^{2}$ floor space per rat). At the beginning of this stage of the study, LE males and females weighed 360-440 g and 230-300 g, respectively, and at the end of the study they weighed 395-495 g and 255-330 g, respectively. The HDH groups of LE rats were housed in 1.2-2.5 times less than the recommended floor space for rats of these weights.

We housed F344 rats in groups of seven for $\mathrm{HDH}$ (21 in ${ }^{2}$ floor space per rat) and in groups of two for LDH ( 72 in $^{2}$ floor space per rat). At the beginning of this stage of the study, F344 males and females weighed 245-280 g and 155-180 g, respectively, and at the end of the study they weighed 260-315 g and 165-195 g, respectively. The HDH groups of F344 rats were housed in 1.1-1.9 times less than the recommended floor space for rats of these weights.

For each strain, there were two cages of HDH males, two cages of $\mathrm{HDH}$ females, one cage of $\mathrm{LDH}$ males and one cage of LDH females. Most experiments at WRAIR/NMRC use rats housed at low densities as described above; therefore, we considered LDH rats to be a control group for comparison with $\mathrm{HDH}$ rats.

\section{Husbandry and management (second stage)}

We housed rats in standard filter-top covered polycarbonate cages ( 144 in $^{2}$ of floor space, 8 in high; Lab Products, Inc., Seaford, DE) on woodchip bedding (Beta Chip, Northeastern Products Corp, Warrensburg, NY). All rats received rodent chow ad libitum (LabDiet RMH3000, PMI Nutrition International, LLC, Brentwood, MO) and drank municipal water, which was filtered by reverse osmosis, through an automatic watering system. For environmental enrichment, we put two plastic pipes (6 in long, 3 in in diameter) in each HDH cage and one plastic pipe in each LDH cage. We maintained cages in a climate-controlled room with temperature set at $18-26^{\circ} \mathrm{C}$ and humidity at $30-70 \%$, with a light:dark cycle of $12 \mathrm{~h}: 12 \mathrm{~h}$.
We maintained rats under these conditions for 4 weeks. We changed cages two times a week for the LDH groups and an average of three times a week for the HDH groups, as needed. We carried out cage changes after completing data collection for the day.

\section{Rat manipulations (second stage)}

We handled and examined all rats for at least $20 \mathrm{~s}$ twice daily. We examined rats for clinical and behavioral signs of stress, including chromodacryorrhea, hair loss, wounds, inappetence, lethargy, ruffled haircoat and hunched appearance. We scored each rat's condition once daily, using a subjective system in which 0 indicated no signs of stress, and 1 indicated that at least one sign was observed. At the end of the study period ( 29 d), we totaled these scores for each rat. We also noted whether any rats required medical treatment, which would result in removal from the study. We weighed each rat once per week (SK-LOK scale, A\&D Co., Ltd, Seoul, Korea).

We collected blood once every 2 weeks at the same time of day (three samplings total; the first sampling was on the last day of the first stage of the study (day 0 ), before rats were regrouped, to evaluate WBC count, blood glucose concentration and serum corticosterone concentration). For blood sampling, we anesthetized rats with 2\% isoflurane using an IMPAC6 anesthesia system (VetEquip, Pleasanton, CA). We collected blood samples $(0.5-1.0 \mathrm{ml})$ from the orbital venous plexus using heparinized hematocrit tubes. For WBC counts and measurement of glucose concentration, we collected blood samples $(0.25-0.5 \mathrm{ml})$ from all rats and stored them in anticoagulant $\left(\mathrm{K}_{2}\right.$-EDTA) microcontainers. We measured glucose concentration immediately after blood collection using a commercial glucometer (ACCU-Check Advantage, Roche, Mannheim, Germany). We obtained the WBC count within $2 \mathrm{~h}$ of blood sampling using a Beckman Coulter AcT Diff hematology analyzer (Beckman Coulter, Miami, FL).

For serum corticosterone analysis, we collected additional blood $(0.25-0.5 \mathrm{ml})$ from at least one rat in each cage ( $n=10$ for each strain) and stored the samples in serum separator microcontainers. We selected this subgroup of rats arbitrarily at the beginning of the study, and we collected blood for corticosterone analysis from the same rats at each sampling session. We froze serum at $-29^{\circ} \mathrm{C}$ and sent it to the Michigan State College of Veterinary Medicine Endocrinology Laboratory for corticosterone analysis.

We considered the measurements recorded on day 0 to be baseline parameters.

\section{Statistics}

We analyzed data separately for each strain, comparing cage groups according to gender and housing density. We analyzed clinical and behavioral signs of stress 

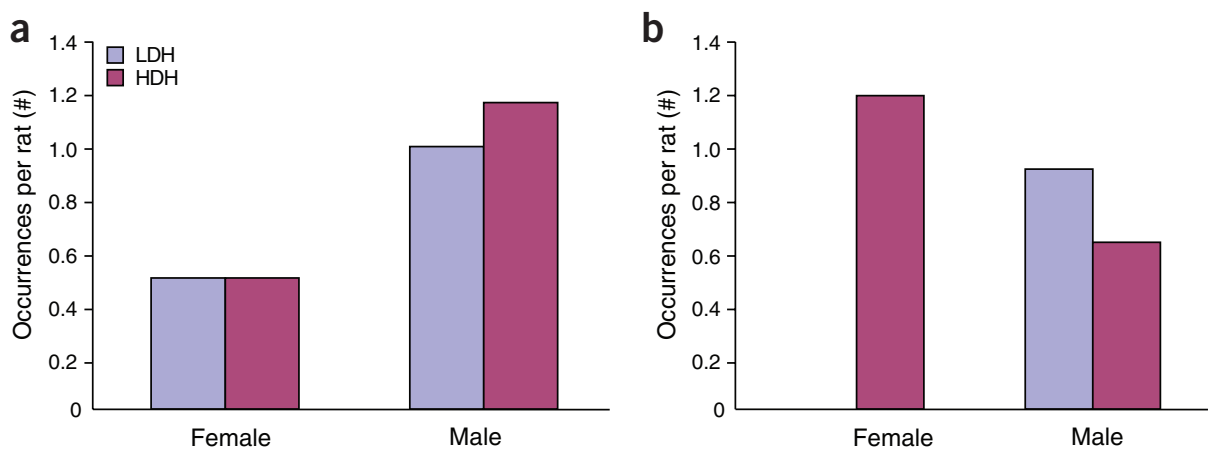

FIGURE 1 | Average number of occurrences per rat of clinical or behavioral signs of stress over a period of $29 \mathrm{~d}$. Rats are separated by gender and by housing density. (a) LE rats. (b) F344 rats.

using Poisson regression. We used ANOVAs to analyze weight change and data obtained from blood samples. As noted above, we analyzed blood glucose concentration and WBC count using three blood samples for each rat. For serum corticosterone concentration, we examined samples taken from 10 rats (at least one rat per cage; three samples per rat). For each parameter measured, we analyzed the difference between data obtained from the first blood sample and from the last. The random term for statistical analyses was the assignment of rats to groups.

\section{RESULTS}

\section{Clinical and behavioral signs of stress}

In LE rats, housing density did not affect the occurrence of clinical and behavioral signs of stress $(P=0.86)$. Gender had a moderate effect on stress $(P=0.057)$; incidence of clinical and behavioral signs was more than two times higher in males than that in females (Fig. 1a).

In F344 rats, the occurrence of clinical and behavioral signs of stress was not affected by cage density $(P=0.29)$ or by gender $(P=0.27)$. The interaction effect between gender and housing density was highly significant $(P<0.0001)$. The incidence of stress signs in LDH males was slightly higher than that in $\mathrm{HDH}$ males, though results were not statistically significant. $\mathrm{HDH}$ females showed a significantly higher incidence of stress signs compared with LDH females $(P<0.0001)$. Over the course of the $29-\mathrm{d}$ study, we noted signs of stress in $\mathrm{HDH}$ females about 1.3 times on average for each rat, whereas in LDH females, we observed no signs of stress (Fig. 1b).

\section{Weight gain}

On the first day of the second stage of the study, the weights of all subjects were within the expected range for rats aged 80-90 d (ref. 28). In LE rats, initial weight was significantly influenced by gender $(P<0.0001$; males were significantly heavier than females) but not by cage density $(P=0.3354)$. At the end of the study, all rats gained weight, but males gained more weight than females did. Weight gain (expressed as a percentage of rats' initial weight) was significantly influenced by both gender $(P<0.0165)$ and cage density $(P=0.0245)$. By the end of the study, LDH males were heavier than $\mathrm{HDH}$ males, whereas $\mathrm{HDH}$ females were heavier than LDH females (Fig. 2a); this interaction effect was not statistically significant, however $(P=0.87)$.
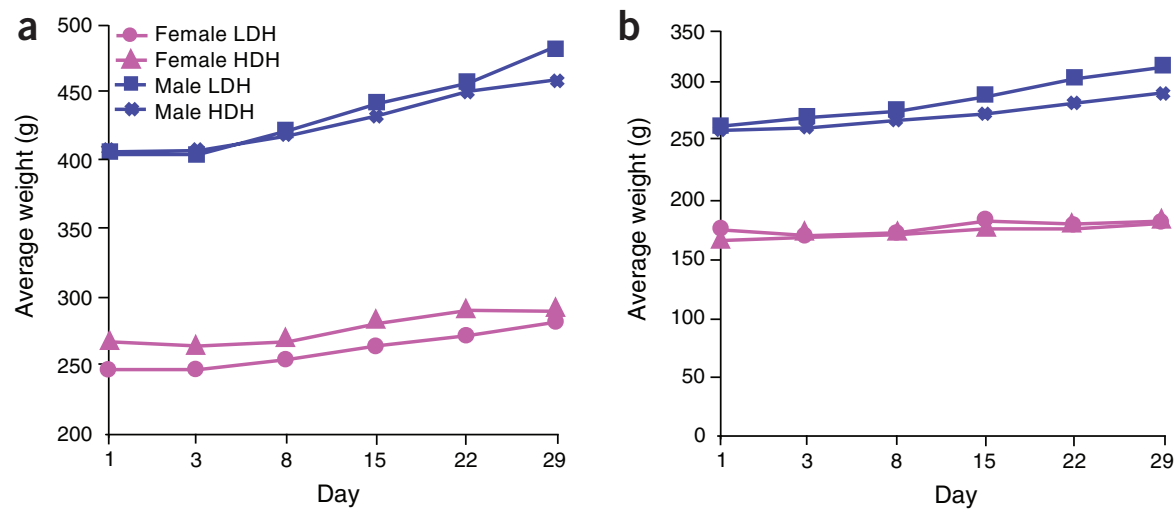

FIGURE 2 | Average weight in male and female rats in LDH and HDH groups. (a) LE rats. (b) F344 rats. 


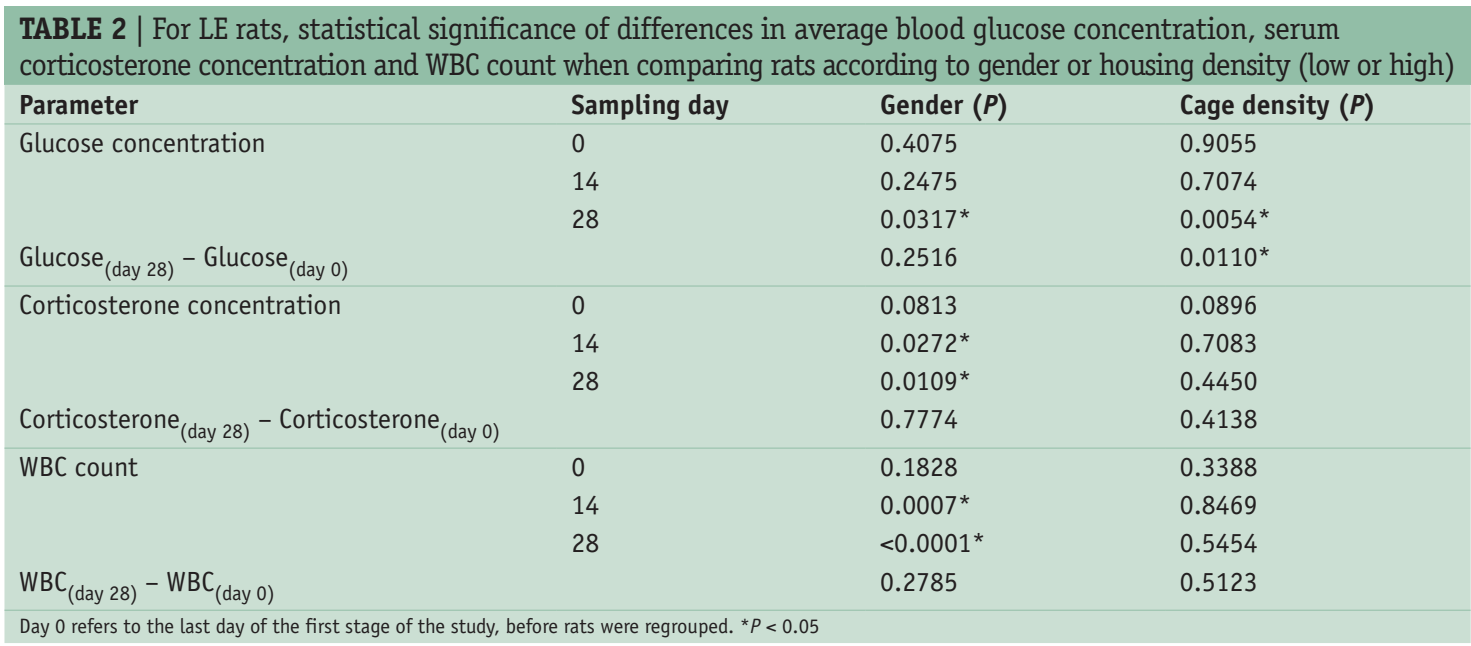

In F344 rats, initial weight was also significantly influenced by gender $(P<0.0001$; males were significantly heavier than females) but not by cage density $(P=0.3189)$. Weight gain was significantly influenced by gender $(P<0.0011)$, with males weighing more than females at the end of the study. We observed a significant interaction effect between cage density and gender for weight gain in this strain, suggesting that $\mathrm{LDH}$ males gained slightly more weight than did $\mathrm{HDH}$ males $(P=0.0043)$. Despite this observation, housing density had no significant effect on rats' overall weight at the end of the study $(P=0.1683$; Fig. $2 \mathbf{b})$.

\section{Physiologic parameters}

We analyzed WBC count, blood glucose concentration and serum corticosterone concentration from blood samples taken at 2-week intervals (three samples per rat). We also compared data from the third blood sample (taken on day 28) with data from the first sample (taken on day 0 ).

In LE rats, at least one parameter for each sampling period was significantly influenced by gender. Gender did not significantly affect the difference between data obtained from the first blood sample and the last. Average blood glucose concentration and WBC were higher in males than in females, whereas corticosterone concentration was lower in males than in females (Tables 2 and 3 ). Cage density significantly affected glucose concentration in the third blood sample, though in the first two samples glucose concentration was similar in HDH and LDH groups (Fig. 3 ). This effect can be attributed to a single LDH rat, in which glucose concentration in the third blood sample was $204 \mathrm{mg} / \mathrm{dl}, 50 \mathrm{mg} / \mathrm{dl}$ more than that in any other rat. The rat's previous two readings were $100 \mathrm{mg} / \mathrm{dl}$ and $132 \mathrm{mg} / \mathrm{dl}$. If this data point is considered an outlier, the difference between the two cage densities is no longer statistically significant $(P=0.25)$.

In F344 rats, gender had some significant effects on all WBC readings and on the last glucose reading. On average, measurements in males were higher than those in females for all physiologic parameters (Tables 4 and 5). Gender had a slight effect on the difference between data from the first and last samples; in females, all measurements decreased, whereas in males, they increased $(P=0.0964)$. Cage density only affected corticosterone concentration (Fig. 4). LDH rats had lower corticosterone concentrations at the second and third readings $(65 \mu \mathrm{g} / \mathrm{dl}$ and $69 \mu \mathrm{g} / \mathrm{dl}$ on average, respectively) compared with $\mathrm{HDH}$ rats $(259 \mu \mathrm{g} / \mathrm{dl}$ and $222 \mu \mathrm{g} / \mathrm{dl}$, respectively $)$.

\section{DISCUSSION}

This was a pilot study to determine whether housing rats at high densities from age 5 weeks through puberty, regrouping them with unfamiliar rats and then continuing to house them at high densities for 4 weeks would alter

\section{TABLE 3 | For LE rats, means and s.d. for physiological parameters with significant differences by gender}

\begin{tabular}{llllll} 
Parameter & Sampling day & \multicolumn{2}{l}{ Female } & \multicolumn{2}{l}{ Male } \\
Glucose $(\mathrm{mg} / \mathrm{dl})$ & & Mean & s.d. & Mean & s.d. \\
Corticosterone $(\mu \mathrm{g} / \mathrm{dl})$ & 28 & 121.1 & 17.5 & 135.8 & 22.1 \\
Corticosterone $(\mu \mathrm{g} / \mathrm{dl})$ & 14 & 510.0 & 141.0 & 283.8 & 73.8 \\
WBC count $\left(10^{-3} \times\right.$ cells $\left./ \mu \mathrm{l}\right)$ & 28 & 648.4 & 163.6 & 367.2 & 72.4 \\
WBC count $\left(10^{-3} \times\right.$ cells $\left./ \mu \mathrm{l}\right)$ & 14 & 5.9 & 1.0 & 8.7 & 2.3 \\
\end{tabular}


behavioral or physiologic parameters, compared with rats that were housed at low densities and were also rehoused with unfamiliar rats after puberty. The Guide currently recommends housing rats of the weights of those used in this study in 23-60 in ${ }^{2}$ of floor space ${ }^{1}$. This recommendation is aimed at preventing stress and aggression, which may occur when rats are housed at higher densities.

To determine whether rats showed signs of stress, aggression or unthriftiness when housed at higher densities, we used a subjective scoring system to evaluate the incidence of common clinical and behavioral signs of stress. Although we observed some signs of stress, neither strain showed a frequency or severity of abnormalities that would indicate that a decreased amount of floor space caused high stress in either gender. We evaluated all clinical signs equally on a 'present or absent' basis but noted that chromodacryorrhea was the most prevalent abnormality (data not shown).

LE males in both housing densities showed more clinical and behavioral signs of stress than did females of this strain. This may indicate that male LE rats are not as compatible as females in group housing situations. Cage density did not have statistically significant effects on the incidence of signs of stress in either gender. In F344 rats, LDH males showed more signs of stress than did HDH males. LDH and HDH males all showed fewer signs of stress than did HDH females. We did not observe signs of stress in F344 LDH females. This may indicate that the females of this strain would not be as compatible as males in high-density group housing situations. None of the observed abnormalities were severe enough to require medical attention or removal from the study.

We observed no overt signs of inappetence in any of the rats. In LE rats, gender and cage density each had significant effects on weight. As noted above, $\mathrm{LDH}$ males weighed more on average than did $\mathrm{HDH}$ males, whereas $\mathrm{HDH}$ females weighed more than LDH females. In F344 rats, cage density did not affect over-

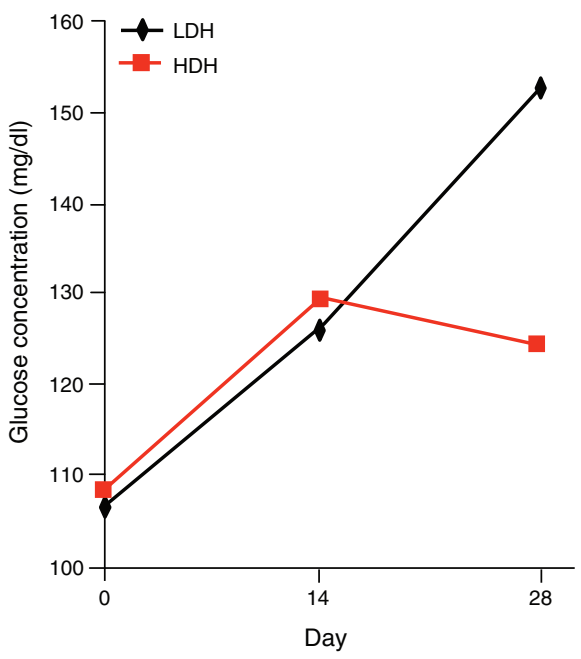

FIGURE 3 | Average glucose concentrations in LE rats. Data from males and females are combined. One male rat in the LDH group had a single reading (on day 28) that was $50 \mathrm{mg} / \mathrm{dl}$ higher than those of any other rat and was markedly higher than other readings for the same rat.

all body weight, though it did affect weight gain; LDH males gained more weight than did HDH males. All rats in both strains gained weight during the study, and their weights were in the accepted range for their ages ${ }^{28}$. We did not submit rats for necropsy and did not measure organ weights. There may have been alterations in the weights of some of the organs, similar to the alterations observed in another group-housing study ${ }^{2}$.

We obtained mixed results when we measured physiological parameters. In LE rats, cage density did not affect WBC or corticosterone concentration, though it did affect glucose concentration. Glucose concentration in LDH males seemed to be higher than that in $\mathrm{HDH}$ males. We note, however, that this observation was based on one reading for a single rat, and this reading

\begin{tabular}{|c|c|c|c|}
\hline Parameter & Sampling day & Gender $(P)$ & Cage density $(P)$ \\
\hline Glucose concentration & 0 & 0.1103 & 0.3906 \\
\hline & 14 & 0.0885 & 0.4924 \\
\hline & 28 & $0.0320 *$ & 0.2125 \\
\hline Glucose $_{\text {(day 28) }}-$ Glucose $_{(\text {day } 0)}$ & & 0.2900 & 0.5284 \\
\hline Corticosterone concentration & 0 & 0.0840 & 0.1381 \\
\hline & 14 & 0.2765 & $0.0239 *$ \\
\hline & 28 & 0.7638 & $0.0529 *$ \\
\hline Corticosterone $_{(\text {day } 28)}-$ Cortico & & 0.0964 & 0.8824 \\
\hline WBC count & 0 & $0.0266^{*}$ & 0.8427 \\
\hline & 14 & $0.0285^{*}$ & 0.4817 \\
\hline & 28 & $0.0004^{*}$ & 0.2967 \\
\hline$W B C_{\text {(day 28) }}-W B C_{\text {(day 0) }}$ & & 0.127 & 0.6216 \\
\hline
\end{tabular}




\begin{tabular}{|c|c|c|c|c|c|}
\hline & Sampling day & Female & & Male & \\
\hline & & Mean & s.d. & Mean & s.d. \\
\hline Glucose (mg/dl) & 28 & 98.5 & 9.66 & 110.56 & 19.37 \\
\hline WBC count $\left(10^{-3} \times\right.$ cells $\left./ \mu \mathrm{l}\right)$ & 0 & 6.41 & 1.45 & 7.82 & 1.87 \\
\hline WBC count $\left(10^{-3} \times\right.$ cells $\left./ \mu \mathrm{l}\right)$ & 14 & 6.75 & 1.95 & 8.40 & 2.06 \\
\hline WBC count $\left(10^{-3} \times\right.$ cells $\left./ \mu \mathrm{l}\right)$ & 28 & 5.03 & 0.64 & 6.79 & 1.64 \\
\hline
\end{tabular}

was much higher than the rat's other two measurements. Therefore, this data point is probably an outlier caused by a lab error or an unrecorded stress event at the time of sampling. If this one value is discounted, none of the parameters indicate a difference in stress level between the two cage densities. In F344 rats, cage density did not affect glucose concentration or WBC. The average corticosterone concentration in LDH rats was lower than that in HDH rats.

According to these results, increasing cage density did not seem to cause adverse physiologic effects in LE rats. F344 rats housed at high densities may have higher short-term stress compared with rats housed at low densities. Although we did not find strain-specific published reference values for all of the physiological parameters we measured, most of the glucose and WBC measurements we obtained were within the accepted normal range for rats ${ }^{29}$. Corticosterone concentrations were also mostly within the published normal ranges, so the increase we observed in HDH F344 rats, which was outside of the normal range, seems to be a true elevation indicating a physiologic stress response. Handling and restraint are known to cause an increase of corticosterone concentration in rats, but as all rats were handled in the same manner, this effect should have been normalized for all groups ${ }^{5}$. Baseline values of physiological parameters may differ for males and females ${ }^{29}$.

We did not collect corticosterone samples from all rats because of processing and logistical constraints. Because this work was carried out as a pilot study, we

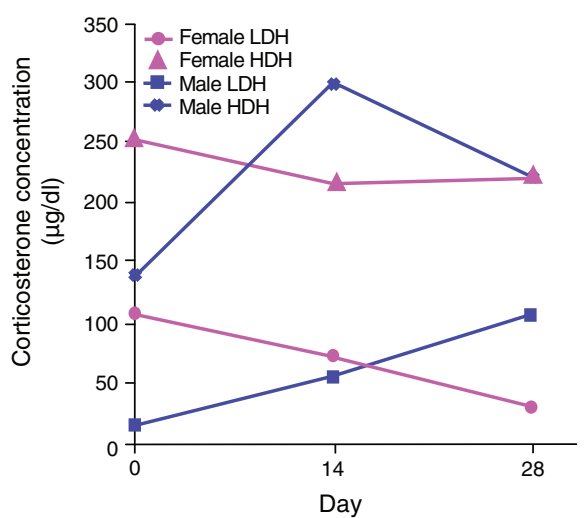

FIGURE 4 | Average corticosterone concentrations in F344 rats. collected a representative serum sample from each cage in order to determine whether any trends could be extrapolated. The differences noted suggest that corticosterone levels should be measured for all rats in future expanded studies.

Because normal husbandry activities can be stressful for animals, the different frequency of cage changes for $\mathrm{LDH}$ and $\mathrm{HDH}$ rats might have been a confounding variable. To minimize this potential effect, we changed all cages after the collection of data. Another variable that might confound comparison between strains is the difference in group size between the HDH groups of each strain. The difference in the number of rats per cage in each strain was based on the differences in the average body weight of rats in these strains. To make comparisons between the strains, it would be beneficial to standardize the group sizes and available floor space per rat. Furthermore, group size may affect rats' behavior regardless of the amount of floor space available. In larger groups of rats, the social hierarchy is more complex than that in smaller groups, which may lead to increased incidence of aggression or stress responses. This variable should be considered when evaluating some of the differences seen between the $\mathrm{HDH}$ and LDH groups within each strain.

\section{CONCLUSIONS}

Our findings suggest that LE and F344 rats that have been housed at high densities from weaning through puberty may be arbitrarily regrouped and housed for at least 4 weeks at densities that are higher than the Guide's current recommendations, without adverse effects on behavioral or physiological parameters. Housing density had little effect on the common indicators of stress that we evaluated (clinical and behavioral signs, weight and physiologic parameters). We did note that in F344 rats, corticosterone concentration was higher in $\mathrm{HDH}$ rats compared with $\mathrm{LDH}$ rats, which may indicate that high-density housing is not optimal for rats of this strain. In addition, gender affected stress in LE rats, whereas in F344 rats it did not. All rats showed normal growth with no clinical signs of severe stress or serious behavioral abnormalities that would result in exclusion from a study. More studies are needed to further investigate potential consequences of transferring post-pubertal group-housed rats into new 
groups, at less floor space per rat than what is recommended in the Guide. Another point for investigation is the effect of housing regrouped rats at high densities for long periods of time.

\section{ACKNOWLEDGMENTS}

This work would not have been possible without the assistance of the WRAIR Malaria Research lab technicians Shannon McGrath and Joseph Shott; Division of Veterinary Medicine technicians Michelle Benford, Theresa Bratton and April Johnson; statistical support from Charles White; and the Endocrinology Laboratory at Michigan State University. This material has been reviewed by WRAIR/NMRC. There is no objection to its presentation or publication. The opinions or assertions contained herein are the private view of the authors and are not to be construed as official or as reflecting the views of WRAIR/NMRC, Department of the Army or the Department of Defense. Research was conducted in compliance with the Animal Welfare Act and other federal statutes and regulations relating to animals and experiments involving animals and adheres to principles stated in the Guide.

\section{COMPETING INTERESTS STATEMENT}

The authors declare no competing financial interests.

\section{Received 25 September 2007; accepted 23 June 2008}

Published online at http://www.labanimal.com/

1. Institute of Laboratory Animal Resources, National Research Council. Guide for the Care and Use of Laboratory Animals (National Academy Press, Washington, DC, 1996).

2. Yildiz, A., Hayirli, A., Okumus, Z., Kaynar, 0. \& Kisa, F. Physiological profile of juvenile rats: effects of cage size and cage density. Lab Anim. (NY) 36, 28-38 (2007).

3. Rock, F.M., Landi, M.S., Hughes, H.C. \& Gagnon, R.C. Effects of caging type and group size on selected physiologic variables in rats. Contemp. Top. Lab. Anim. Sci. 36, 69-72 (1997).

4. Smith, A.L., Mabus, S.L., Muir, C. \& Woo, Y. Effects of housing density and cage floor space on three strains of young adult inbred mice. Comp. Med. 55, 368-376 (2005).

5. Vachon, P. \& Moreau, J.P. Serum corticosterone and blood glucose in rats after two jugular vein blood sampling methods: comparison of the stress response. Contemp. Top. Lab. Anim. Sci. 40, 22-24 (2001).

6. Krohn, T.C., Sorensen, D.B., Ottesen, J.L. \& Hansen, A.K. The effects of individual housing on mice and rats: a review. Anim. Welfare 15, 343-352 (2006).

7. Salvin, S.B., Rabin, B.S. \& Neta, R. Evaluation of immunologic assays to determine the effects of differential housing on immune reactivity. Brain Behav. Immun. 4, 180-188 (1990).

8. Sharp, J., Zammit, T., Azar, T. \& Lawson, D. Stress-like responses to common procedures in individually and grouphoused female rats. Contemp. Top. Lab. Anim. Sci. 42, 9-18 (2003).

9. Viveros, M.P., Hernandez, R., Martinez, I. \& Gonzalez, P. Effects of social isolation and crowding upon adrenocortical reactivity and behavior in the rat. Rev. Esp. Fisiol. 44, 315-321 (1988).

10. Sharp, J., Azar, T. \& Lawson, D. Does cage size affect heart rate and blood pressure of male rats at rest or after procedures that induce stress-like responses? Contemp. Top. Lab. Anim. Sci. 42, 8-13 (2003).

11. Sharp, J.L., Zammit, T.G., Azar, T.A. \& Lawson, T.M. Stress-like responses to common procedures in male rats housed alone or with other rats. Contemp. Top. Lab. Anim. Sci. 41, 8-14 (2002).

12. Armario, A., Ortiz, R. \& Balasch, J. Effects of crowding on some physiological and behavioral variables in adult male rats. Physiol. Behav. 32, 35-37 (1984).

13. Sharp, J., Zammit, T., Azar, T. \& Lawson, D. Does witnessing experimental procedures produce stress in male rats? Contemp. Top. Lab. Anim. Sci. 41, 8-12 (2002).

14. Duke, J.L., Zammit, T.G. \& Lawson, D.M. The effects of routine cage-changing on cardiovascular and behavioral parameters in male Spraque-Dawley rats. Contemp. Top. Lab. Anim. Sci. 40, 17-20 (2001).

15. van den Buuse, M., Van Acker, S.A., Fluttert, M. \& De Kloet, E.R. Blood pressure, heart rate, and behavioral responses to psychological "novelty" stress in freely moving rats. Psychophysiology 38, 490-499 (2001).

16. Fagin, K.D., Shinsako, J. \& Dallman, M.F. Effects of housing and chronic cannulation on plasma ACTH and corticosterone in the rat. Am. J. Physiol. 245, 515-520 (1983).

17. Brown, K.J. \& Grunberg, N.E. Effects of housing on male and female rats: crowding stresses male but calm females. Physiol. Behav. 58, 1085-1089 (1995).

18. Sharp, J., Zammit, T., Azar, T. \& Lawson, D. Are "by-stander" female Sprague-Dawley rats affected by experimental procedures? Contemp. Top. Lab. Anim. Sci. 42, 19-27 (2003).

19. Hurst, J.L, Barnard, C.J, Tolladay, U., Nevision, C.M. \& West, C.D. Housing and welfare in laboratory rats: effects of cage stocking density and behavioural predictors of welfare. Anim. Behav. 58, 563-586 (1999).

20. Enoka, R.M., Rankin, L.L. \& Stuart, D.G. Cage-size and gender effects on fatigue in rats. Physiologist 28 Suppl, S137-S138 (1985).

21. Pellis, S.M. \& Pellis, V.C. Role reversal changes during the ontogeny of play fighting in male rats: Attack vs defense. Aggressive Behav. 17, 179-189 (1991).

22. Lore, R., Nikoletseas, M. \& Takahashi, L. Colony aggression in laboratory rats: a review and some recommendations. Aggressive Behav. 10, 59-71 (1984).

23. Henry, J.P. Biological basis of the stress response. Integr. Physiol. Behav. Sci. 27, 66-83 (1992).

24. Meaney, M.J. \& Stewart, J. A descriptive study of social development in the rat (Rattus norvegicus). Anim. Behav. 29, 34-45 (1981).

25. Takahashi, L.K. \& Lore, R.K. Intermale and maternal aggression in adult rats tested at different ages. Physiol. Behav. 29, 1013-1018 (1982).

26. Takahashi, L.K. \& Lore, R.K. Play-fighting and the development of agonistic behavior in male and female rats. Aggressive Behav. 9, 217-227 (1983).

27. Arakawa, H. Age dependent effects of space limitation and social tension on open-field behavior in male rats. Physiol. Behav. 84, 429-436 (2005).

28. Charles River Laboratories. Charles River Laboratories Research Models and Services 6, 9 (Charles River Laboratories, Wilmington, MA, 2006).

29. Kohn, D.F. \& Clifford, C.B. in Laboratory Animal Medicine 2nd edn. (eds. Fox, J.G., Anderson, L.C., Loew, F.M. \& Quimby, F.W.) 127-131 (Academic, San Diego, 2002). 